\title{
Polarization dependence of linewidth enhancement factor in InGaAs/InGaAsP MQW material
}

Jepsen, Kim Stokholm; Storkfelt, Niels; Vaa, Michael; Stubkjær, Kristian

Published in:

I E E E Journal of Quantum Electronics

Link to article, DOI:

10.1109/3.286149

Publication date:

1994

Document Version

Publisher's PDF, also known as Version of record

Link back to DTU Orbit

Citation (APA):

Jepsen, K. S., Storkfelt, N., Vaa, M., \& Stubkjær, K. (1994). Polarization dependence of linewidth enhancement factor in InGaAs/InGaAsP MQW material. I E E E Journal of Quantum Electronics, 30(3), 635-639.

https://doi.org/10.1109/3.286149

\section{General rights}

Copyright and moral rights for the publications made accessible in the public portal are retained by the authors and/or other copyright owners and it is a condition of accessing publications that users recognise and abide by the legal requirements associated with these rights.

- Users may download and print one copy of any publication from the public portal for the purpose of private study or research.

- You may not further distribute the material or use it for any profit-making activity or commercial gain

- You may freely distribute the URL identifying the publication in the public portal 


\title{
Polarization Dependence of Linewidth Enhancement Factor in InGaAs/InGaAsP MQW Material
}

\author{
Kim S. Jepsen, Niels Storkfelt, Student Member, IEEE Michael Vaa, and Kristian E. Stubkjaer, Member, IEEE
}

\begin{abstract}
Measurements and calculations on the differential gain, the differential refractive index, and the linewidth enhancement factor have been performed for unstrained quantum-well (QW) material. The differential refractive index is considerably lower for the transverse magnetic (TM) polarization than for the transverse electric (TE) polarization, which is ascribed to absence of the plasma effect for the TM polarization. This has implications for the linewidth enhancement factor and thus linewidth and chirp in $\mathbf{Q W}$ lasers.
\end{abstract}

\section{INTRODUCTION}

O PTICAL devices based on multiple quantum-well (MQW) structures are interesting components because of the improved performance compared to conventional structures. Material parameters such as the linewidth enhancement factor (known as the $\alpha$-factor) and the related differential gain and differential refractive index are important device parameters because they govern properties such as linewidth and chirp [1], [2], resonance frequency, and frequency response. Because of the inherent anisotropy of QW structures, these parameters depend on the polarization, and thus knowledge of the polarization dependence is important for optimization of the devices. In this paper, measured and calculated results for the polarization dependence of the $\alpha$ factor, the differential gain, and the differential refractive index are presented for a 4-well MQW semiconductor optical amplifier (SOA). It is found that the differential refractive index for small injection currents is considerably smaller for the TM-polarization than for the TE-polarization. This is ascribed to the absence of the plasma effect for the TMpolarization, owing to the transverse confinement of the carriers in the well [3], [4].

\section{THEORY}

To evaluate the differential gain and the contribution to the differential refractive index due to anomalous dispersion, the gain spectrum must be calculated. This is done using the $\mathbf{k} \cdot \mathbf{p}$ approximation, taking into account both the heavy and light hole band, and assuming parabolic dispersion; the model is described in [5]. However, in comparison with [5], the present model includes carrier overflow in a simple way, assuming a common quasi-Fermi-level in both the $\mathrm{QW}$ and

Manuscript received March, 19, 1993; revised May 31, 1993.

The authors are with the Center for Broadband Telecommunications, Electromagnetics Institute, Technical University of Denmark, DK-2800 Lyngby, Denmark.

IEEE Log Number 9215354. the SCH regions for both electrons and holes [6]. For the sake of simplicity, charge neutrality has been assumed for the structure as a whole ( $\mathrm{SCH}$ and wells) in contrast to the more precise assumption of local charge neutrality [7]. This implies that the number of electrons is not equal to the number of holes in the QW states, and thus both the differential gain and the differential refractive index can be defined with respect to either the electron or the hole density. In this paper the differential gain and the differential refractive index are defined with respect to the hole density, as explained below.

The measurement of the differential gain and the differential refractive index is performed by measuring the amplitude modulation (AM) and phase modulation (PM) index obtained by modulating the carrier density through modulation of the bias current [8]. The AM index is given by:

$$
\begin{aligned}
m & =\frac{1}{2} L \delta g \\
& =\frac{1}{2} L\left(\left(\frac{\partial g_{m}}{\partial N}\right)_{P=\text { const }} \cdot \frac{\delta N}{\delta I}+\left(\frac{\partial g_{m}}{\partial P}\right)_{N=\text { const }} \cdot \frac{\delta P}{\delta I}\right) \delta I
\end{aligned}
$$

where $m$ is the AM index, $L$ the length of the amplifier, $\partial g_{m} /\left.\partial N\right|_{P}$ the differential modal gain with respect to the electron density $N$ in the well at constant hole density $P$, and $\partial g_{m} /\left.\partial P\right|_{N}$ vice versa. $g_{m}=\Gamma g-\alpha_{\text {loss}}$, where $g_{m}$ is the modal gain, $\Gamma$ the confinement factor, $g$ the material gain and $\alpha_{\text {loss }}$ the internal losses; finally $\delta I$ is the amplitude of the current modulation. Using small-signal analysis it can be shown that $m$ can be expressed in the two equivalent forms (assuming that only the electrons leak significantly over the barrier):

$$
\begin{aligned}
m & =\frac{1}{2} L \frac{d g_{m}}{d N} \frac{\delta I}{q V} \frac{\tau_{d, N}}{\sqrt{1+\chi^{2}\left(\omega \tau_{d, N}\right)^{2}}} \\
& =\frac{1}{2} L \frac{d g_{m}}{d P} \frac{\delta I}{q V} \frac{\tau_{d, p}}{\sqrt{1+\left(\omega \tau_{d, P}\right)^{2}}}
\end{aligned}
$$

where $q$ is the unit charge, $V$ the active volume, $\omega$ the angular frequency of the modulation, $\chi \equiv d P / d N, \tau_{d, N}=$ $\left(d R_{\mathrm{sp}} / d N+d R_{\mathrm{stim}} / d N\right)^{-1}$ the differential carrier lifetime with respect to the electron density ( $R_{\mathrm{sp}}$ and $R_{\text {stim }}$ being the total spontaneous and the stimulated recombination rate per unit volume, respectively), and $\tau_{d, P}=\left(d R_{\mathrm{sp}} / d P+\right.$ $\left.d R_{\text {stim }} / d P\right)^{-1}$ the differential carrier lifetime with respect to the hole density. The spontaneous recombination rate is given by $R_{\mathrm{sp}}=A P+B N P+C N P^{2}$ (assuming that the CHHS Auger process is dominant). Equation (2) shows that in the presence of carrier overflow the differential carrier lifetime of 
the holes in the well can be found as $\omega^{-1}$ by measuring the frequency at which the AM index has dropped $1.5 \mathrm{~dB}$ below its zero-frequency value. Knowing the hole differential carrier lifetime, the differential gain with respect to the hole density can be found by measuring the AM index at a given frequency.

The anomalous dispersion contribution to the effective (modal) differential refractive index is found from the Kramers-Kronig transform of the modal differential gain spectrum [9].

$$
\left.\frac{d n_{\mathrm{eff}}(E)}{d P}\right|_{\mathrm{anom}}=-\frac{h c_{0}}{2 \pi^{2}} \int_{0}^{\infty} \frac{d g_{m}(\epsilon) / d P}{(\epsilon-E)(\epsilon+E)} d \epsilon
$$

where $n_{\text {eff }}$ is the effective refractive index, $P$ the density of holes in the well, $h$ Planck's constant, $c_{0}$ the speed of light in vacuum and $E$ the photon energy.

A second contribution to $d n / d P$ is due to the plasma effect [9], in which free carriers responding to the (optical) electric field induce an additional polarization, which acts to reduce the refractive index proportional to the carrier density. For each type of carrier (e.g., electrons in the $\mathrm{SCH}$ region) the change in differential refractive index due to the plasma effect is approximated as:

$$
\left.\Delta n\right|_{\text {plasma }}=-\frac{q^{2} \lambda^{2} N_{i}}{8 \pi^{2} \epsilon_{0} n_{\text {eff }, 0} c_{0}^{2} m_{i}^{*}}
$$

where $q$ is the unit charge, $\lambda$ the wavelength, $\epsilon_{0}$ the vacuum permittivity, $n_{\mathrm{eff}, 0}$ the effective (modal) index in the absence of free carriers, $m_{i}^{*}$ the effective mass and $N_{i}$ the carrier density of the appropriate type of carrier: $N, P, N_{\mathrm{SCH}}$, or $P_{\mathrm{SCH}}$, where $N$ and $P$ is the electron density respective to the hole density in the wells, and $N_{\mathrm{SCH}}$ and $P_{\mathrm{SCH}}$ is the electron density respective the hole density in the SCH-region. For TM-polarized light it is important to note that, since the carriers confined in the wells are not free to move in response to the electric field of the light (in "classical" terms), they do not produce a significant polarization, and the free-carrier plasma effect should therefore be absent for these carriers (Fig. 1). In quantum mechanical terms there are no states (corresponding to $E\left(\mathbf{k}_{\perp}+\mathrm{d} \mathbf{k}_{\perp}\right)$ in bulk material) to which the confined electrons or holes can be scattered by light polarized perpendicularly to the well, i.e., there is no intra-subband scattering. Instead, a skewing of the QW envelope function will occur, but the polarization induced by this effect should be small. For the TE polarization the movement of the confined carriers in the direction of the electric field is not restricted, and the plasma effect will contribute to the differential refractive index. Thus, the total modal differential refractive index is given by:

$$
\begin{aligned}
\frac{d n_{\mathrm{eff}}}{d P}= & \left.\frac{d n_{\mathrm{eff}}}{d P}\right|_{\mathrm{anom}}+\left.\frac{d n_{\mathrm{eff}}}{d P}\right|_{\mathrm{plasma}} \\
= & -\frac{q^{2} \lambda^{2}}{8 \pi^{2} \epsilon_{0} n_{\mathrm{eff}} c_{0}^{2}}\left(\frac{\Gamma_{\mathrm{SCH}, \mathrm{TE}}}{m_{\mathrm{c}}} \frac{d N_{\mathrm{SCH}}}{d P}\right. \\
& +\frac{\Gamma_{\mathrm{SCH}, \mathrm{TE}}}{m_{\nu r}} \frac{d P_{\mathrm{SCH}}}{d P} \\
& \left.+\frac{\Gamma_{\mathrm{TE}}}{m_{c}} \frac{d N}{d P}+\frac{\Gamma_{\mathrm{TE}}}{m_{\nu h}} \frac{d P}{d P}\right)
\end{aligned}
$$

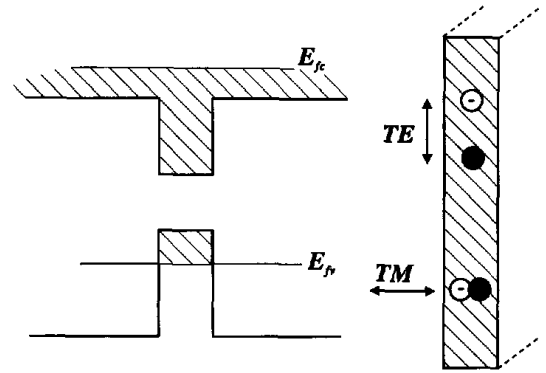

(a)

(b)

Fig. 1. Influence of polarization on the plasma-effect contribution to the differential refractive index. (a) Spatial band structure. (b) Carriers confined in the wells do not contribute to the plasma effect for the TM polarization as they are not free to move in the transverse direction.

$$
\begin{aligned}
\left.\frac{d n_{\mathrm{eff}}}{d P}\right|_{\text {plasma }, \mathrm{TM}}= & -\frac{q^{2} \lambda^{2}}{8 \pi^{2} \epsilon_{0} n_{\mathrm{eff}} c_{0}^{2}}\left(\frac{\Gamma_{\mathrm{SCH}, \mathrm{TM}}}{m_{c}} \frac{d N_{\mathrm{SCH}}}{d P}\right. \\
& \left.+\frac{\Gamma_{\mathrm{SCH}, \mathrm{TM}}}{m_{\nu r}} \frac{d P_{\mathrm{SCH}}}{d P}\right)
\end{aligned}
$$

where $m_{c}$ is the effective mass of the conduction band electrons, $m_{\nu r}=\left(m_{\nu h}^{3 / 2}+m_{\nu l}^{3 / 2}\right) /\left(m_{\nu h}^{1 / 2}+m_{\nu l}^{1 / 2}\right)$ where $m_{\nu h}$ and $m_{\nu l}$ are the effective masses of the heavy and light holes respectively; the effective masses are taken as appropriate for either the SCH or the QW regions. Finally, $\Gamma_{\mathrm{SCH}, \mathrm{TE}}, \Gamma_{\mathrm{SCH}, \mathrm{TM}}$, and $\Gamma_{\mathrm{TE}}$ are the confinement factors (TE- or TM-mode) for the SCH-regions and the active regions, respectively. Having found the modal differential gain $\left(d g_{m} / d P\right)$ and the effective (modal) differential refractive index $d n_{\text {eff }} / d P$, the $\alpha$-factor is simply expressed as [3] $\alpha=$ $-(4 \pi / \lambda) \cdot\left(d n_{\text {eff }} / d P\right) /\left(d g_{m} / d P\right)$.

\section{Results AND Discussion}

The device used in the experiments is a MQW DC-PBH SOA. The four InGaAs wells ( $E_{g}=0.75 \mathrm{eV}$ ) and three InGaAsP barrier layers $\left(E_{g}=1.078 \mathrm{eV}\right)$ are each 80 and 130 $\AA$ wide, respectively. The guide layers ( $\mathrm{SCH}$ ) are each 1000 $\AA$ wide, and of the same material as the barrier layers; all layers are lattice matched to InP. The lateral width of the active stripe is $2 \mu \mathrm{m}$, and the length of the device $800 \mu \mathrm{m}$. The residual facet reflectivity is approximately $10^{-3}$. The TEand TM-polarizations are considered at the respective gain peaks of 1532 and $1500 \mathrm{~nm} ; n_{\text {eff }} \simeq 3.21$ for both TE and TM polarization at these wavelengths.

The calculations are performed using the following parameters: $m_{c}=0.041 m_{0}$ for InGaAs (wells), $m_{c}=0.064 m_{0}$ for InGaAsP (barriers, $\mathrm{SCH}$ ), $m_{\nu h}=0.44 m_{0}$ and $m_{\nu l}=0.055$ $m_{0}$ for both wells and barriers. The depth of the conduction band well has been assumed to be $40 \%$ of the difference between the band gaps of InGaAs and InGaAsP. The band gap shrinkage due to thermal effects and the presence of free carriers has been taken into account. In order to obtain a correct fit to the measured values of the single-pass gain the intraband relaxation time is assumed to vary with the carrier density: $\tau$ (seconds) $=2.70 \cdot 10^{14} \cdot \exp \left(-5.64 \cdot 10^{-26} \mathrm{P}\right)$, in qualitative agreement with [10]. The current is found assuming negligible 


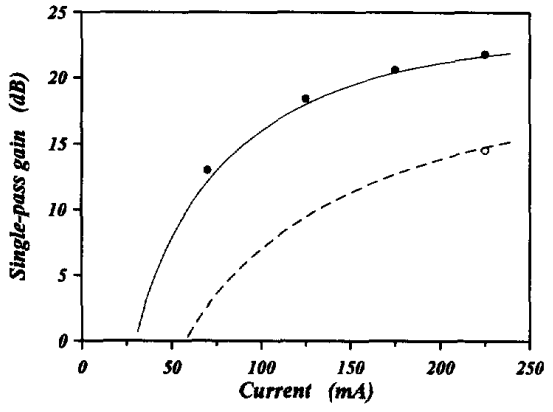

Fig. 2. Measured and calculated single-pass gain versus injection current: TE at $1532 \mathrm{~nm}(\bullet,-)$ and TM at $1500 \mathrm{~nm}(\mathrm{O}, \ldots)$.

recombination from the $\mathrm{SCH}$ and barrier regions $\left(P_{\mathrm{SCH}} \simeq 0\right)$. It is assumed that the stimulated recombinations have only little influence on the differential carrier lifetime, which is a good approximation for optical amplifiers operated well below saturation. Finally, the confinement factors are calculated to have the values $\Gamma_{\mathrm{SCH}, \mathrm{TE}} \simeq 0.422(\lambda=1532 \mathrm{~nm}), \Gamma_{\mathrm{SCH}, \mathrm{TM}} \simeq$ $0.400(2=1500 \mathrm{~nm})$ and $\Gamma_{\mathrm{TE}} \simeq 0.05$.

Measured and calculated results for the single-pass gain at the respective gain peaks are shown in Fig. 2 for the two polarizations. The heating of the junction due to the current has been included in the calculations. A good agreement between measurements and calculations is found, and together with the results obtained in [5] this is taken as a validation of the gain model. Specifically, the difference between the two polarizations seems to be correctly accounted for.

Measured results are obtained for the modal differential gain. Shown in Fig. 3 is the modal gain divided by the appropriate confinement factor, giving the "material" differential gain. Again, a reasonable agreement is obtained. As compared with [4], it is clear that the carrier overflow acts to reduce the differential gain because of the clamping of the conduction band quasi-Fermi level near the top of the conduction band well. It is also notable that while the singlepass gain is significantly smaller for the TM-polarization than for the TE-polarization, this is not the case for the differential gain; in fact, $d g / d P$ is larger for the TM-polarization at high injection currents. This can be explained as follows. First, even though the matrix-element for conduction band-heavy hole band $(c-h h)$ transitions is much smaller for TM-polarized light than for the TE-polarized light (photon energies near the band gap), the situation is completely reversed for the conduction band-light hole band $c-l h$ transitions, as shown in Fig. 4 . However, at low and medium injection currents the main part of the hole density is heavy holes since the light holes have energies in the Boltzmann tail of the Fermi-distribution; thus the magnitude of the TM-gain is small $\left(E_{\perp l h} \simeq E_{\perp h h}+\right.$ $2 k_{B} T$ ). This is not the case for the differential gain since the important factor here is the influence on the gain of the change of the quasi-Fermi levels. Again, since the light holes are found in the Boltzmann tail of the Fermi distribution, an increase of the Fermi level will lead to an exponential increase of the $l h$ Fermi occupation number $f_{\nu l h}$, as is also the case for the $h h$ Fermi occupation number. Because of the large

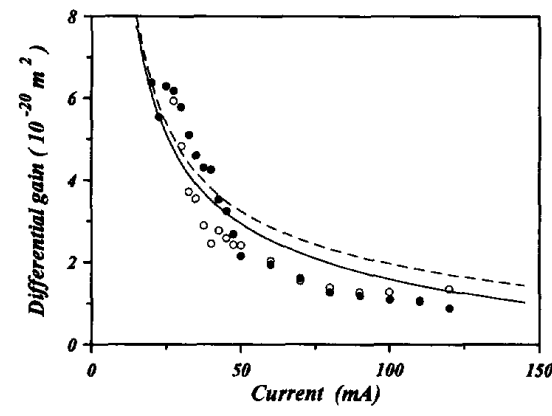

Fig. 3. Measured and calculated results for the differential gain versus injection current for $\mathrm{TE}$ at $1532 \mathrm{~nm}(\bullet,-)$ and $\mathrm{TM}$ at $1500 \mathrm{~nm}(\mathrm{O}$, - -).

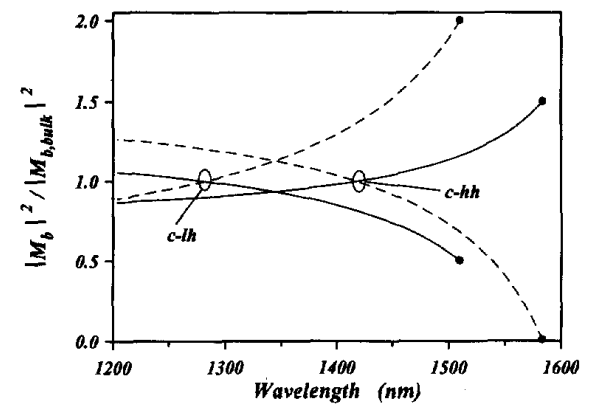

Fig. 4. Quantum well matrix-element (normalized to bulk matrix element) versus wavelength for TE (-) and TM (- - ) polarizations. Effective band gap taken at $2 \mathrm{~mA}$.

matrix element, $d g /\left.d P\right|_{\mathrm{TM}}$ is nearly equal to $d g /\left.d P\right|_{\mathrm{TE}}$ at low currents, and is in fact bigger than $d g /\left.d P\right|_{\mathrm{TE}}$ at higher currents, as seen in both measurements and calculations.

The measured values for the differential refractive index are shown in Fig. 5, together with the calculated results. Again the results are normalized to the active region by division with $\Gamma_{\mathrm{TE}}$ or $\Gamma_{\mathrm{TM}}$, giving the "material" differential refractive index. The measurements show that $d n / d P$ is significantly smaller for the TM polarization than for the TE polarization for low injection currents; the difference, however, vanishes as the current is increased. The dip in the measured values of $d n / d P$ at low bias currents is at present unaccounted for, but might be due to experimental factors. Fig. 6 shows for both polarizations the calculated contributions to $d n / d P$ from the different effects, giving the total differential refractive index. According to the calculations, the difference in $d n / d P$ for the TE and TM polarization cannot be accounted for if the contribution from the plasma effect is equal for the two polarizations; if this were the case $d n / d P$ should actually be higher for the TM polarization. The fact that $d n /\left.d P\right|_{\mathrm{TM}}$ approaches $d n /\left.d P\right|_{\mathrm{TE}}$ at higher currents can be seen from (5). For the TM polarization, the contribution from the electrons in the $\mathrm{SCH}$ and barrier regions increases with the current due to the factor $d N_{\mathrm{SCH}} / d P$. For the TE polarization, this increase is counteracted by the relative decrease of electrons in the well due to the factor $d N / d P$. (Note that $N+N_{\mathrm{SCH}}=$ 


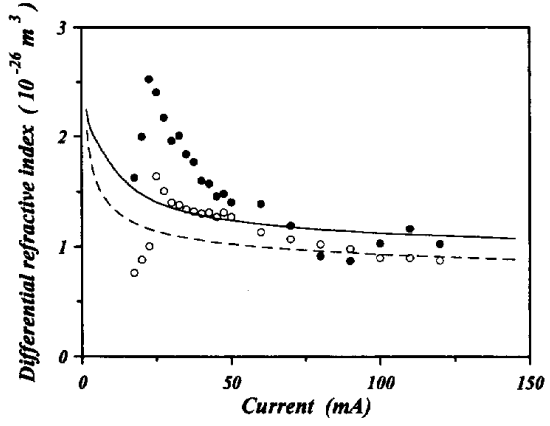

Fig. 5. Measured and calculated results for the differential refractive index versus injection current for TE at $1532 \mathrm{~nm}\left(\bullet,-{ }_{-}\right)$and TM at $1500 \mathrm{~nm}$ $(\mathrm{O},-\cdots)$.

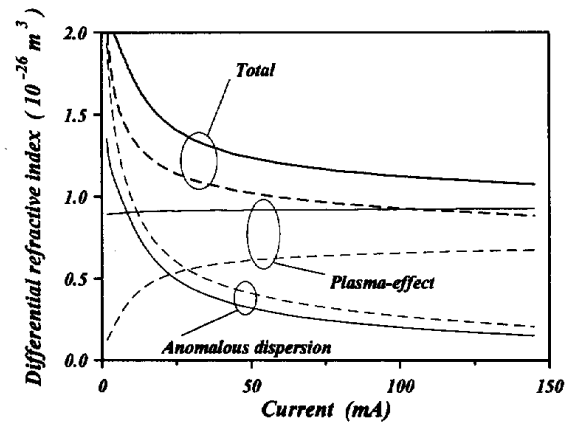

Fig. 6. Contributions to differential refractive index from anomalous dispersion and plasma effect for TE (-) and TM (- - ) polarizations. The contribution from the plasma effect for the TM polarization increases with the current.

$P+P_{\mathrm{SCH}} \simeq P$ ). From Fig. 6 it is clear that the contribution from the plasma effect increases with increasing current for the TM polarization, while this is not the case for the TE polarization. This accounts for the observed behavior of the differential refractive index.

The resulting $\alpha$-factors for the TE and TM polarization are shown versus current in Fig. 7. At low injection currents the $\alpha$-factors are significantly lower for the TM polarization than for the TE-polarization. This is ascribed to the absence of the plasma effect. At medium and high injection currents there is generally no difference between the $\alpha$-factors of the two polarizations; as described above this is probably due to the carrier overflow, which leads to a significant electron population in the $\mathrm{SCH}$ region.

The low $\alpha$-factor for the TM polarization indicates that tensile strained (TS) QW lasers, which oscillate in the TM mode if the strain is sufficient, might have an improved performance with regard to linewidth and chirp in comparison with either unstrained or compressively strained QW lasers. Low linewidths and $\alpha$-factors have actually been reported previously [3], [11] for TS QW lasers. A further benefit in this connection is that the TM mode couples better to the grating of DFB and DBR lasers, thereby improving the tendency for single-mode operation. It should be noted, however, that

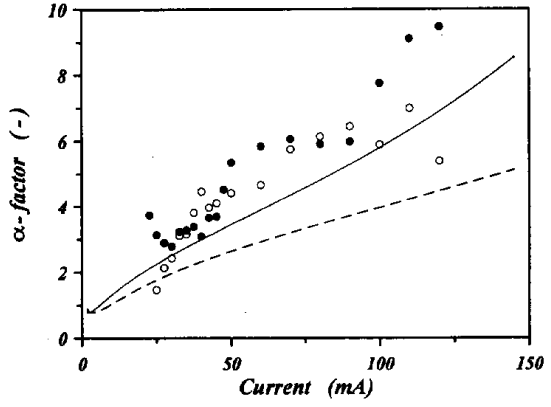

Fig. 7. Measured and calculated results for the linewidth enhancement factor versus injection current: TE at $1532 \mathrm{~nm}(\bullet, \longrightarrow), \mathrm{TM}$ at $1500 \mathrm{~nm}(\mathrm{O},---)$.

the band offsets in InGaAs/InGaAsP TS QW structures are such that the conduction band well is very shallow [12]. Carrier overflow might therefore be significant, which would tend to increase the differential refractive index for the TM polarization due to an increased contribution from the plasma effect in the SCH layers. If carrier overflow does indeed pose a serious problem, a material system such as InGaAs/InAlGaAs might be considered, since this system has a deeper conduction band well.

\section{CONCLUSION}

The polarization dependence of the $\alpha$-factor, the differential gain, and the differential refractive index have been investigated experimentally and theoretically for unstrained MQW material. At low injection levels the differential refractive index and the $\alpha$-factor are smaller for the TM polarization than for the TE polarization. The theoretical analysis indicates that this is due to the absence of the plasma effect contribution to the differential refractive index for the TM polarization in QW structures.

\section{ACKNOWLEDGMENT}

Dr. M. Yamaguchi, NEC Opto-electronic Device Research Division, is gratefully acknowledged for supplying the devices used in the experiments.

\section{REFERENCES}

[1] C. H. Henry, "Theory of the linewidth of semiconductor lasers," IEEE J. Quantum Electron., vol. 18, no. 2, pp. 259-264, February 1982.

[2] T. L. Koch and J. E. Bowers, "Nature of wavelength chirping in directly modulated semiconductor lasers," Electr. Lett., vol. 20, no. 25/26, pp. 1038-1039, December 1984.

[3] L. F. Tiemeijer, P. J. A. Thijs, J. J. M. Binsma, and T. v. Dongen, "Effect of free carriers on the linewidth enhancement factor of InGaAs/InP (strained layer) multiple quantum well lasers," Appl. Phys. Lett., vol. 60, no. 20, pp. 2466-2468, May 1992.

[4] M. Soerensen, N. Storkfelt, K. S. Jepsen, and K. E. Stubkjaer, "Polarisation dependent $\alpha$-factor in InGaAs/InGaAsP MQW material," Conf. Dig. 13th IEEE Int'l. Semicond. Laser Conf., Takamatsu, Japan, 1992, paper D-14.

[5] K. S. Jepsen, M. Mikkelsen, J. H. Povlsen, M. Yamaguchi, and K. E. Stubkjaer, "Wavelength dependence of noise figure in InGaAs/InGaAsP multiple-quantum-well laser amplifier," IEEE Photon. Technol. Lett., vol. 4, no. 6, pp. 550-553, June 1992.

[6] R. Nagarajan, T. Kamiya, and A. Kurobe, "Band filling in GaAs/AlGaAs multiquantum well lasers and its effect on the threshold current," IEEE J. Quantum Electron., vol. 25, no. 6, pp. 1161-1170, June 1989. 
[7] N. Tessler, R. Nagar, D. Abraham, and G. Eisenstein, "Coupling between barrier and quantum well energy states in a multiple quantum well optical amplifier," Appl. Phys. Lett., vol. 60, no. 6, pp. 665-667, February 1992.

[8] N. Storkfelt, B. Mikkelsen, D. S. Olesen, M. Yamaguchi, and K. E. Stubkjaer, "Measurement of carrier lifetime and linewidth enhancement factor for 1.5- $\mu \mathrm{m}$ ridge-waveguide laser amplifier," IEEE Photon. Technol. Lett., vol. 3, no. 7, pp. 632-634, July 1991.

[9] C. H. Henbry, R. A. Logan, and K. A. Bertness, "Spectral dependence of the change in refractive index due to carrier injection in GaAs lasers," J. Appl. Phys., vol. S2, no. 7, pp. 4457-4461, July 1981.

[10] M. Asada, "Intraband relaxation times in quantum-well lasers," IEEE J. Quantum Electron., vol. 25, no. 9, pp. 2019-2026, September 1989.

[11] P. J. A. Thijs, L. F. Tiemeijer, P. I. Kuindersma, J. J. M. Binsma, and T. v. Dongen, "High performance $1.5 \mu \mathrm{m}$ wavelength InGaAs/InGaAsP strained quantum well lasers and amplifiers," IEEE J. Quantum Electron., vol. 27, no. 6, pp. 1426-1439, June 1991.

[12] G. Jones and E. P. O'Reilly, "Improved performance in tensile strained long wavelength lasers," Conf. Dig. 13th IEEE Int'l. Semicond. Laser Conf., Takamatsu, Japan, 1992, paper C-5.

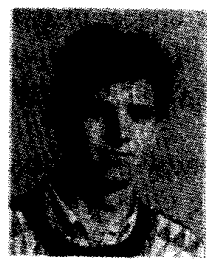

Kim S. Jepsen was born in Copenhagen, Denmark, in December 1963. He received the M.Sc. degree in electrical engineering from the Electromagnetics Institute, Technical University of Denmark (DTU), Lyngby, in 1991.

$\mathrm{He}$ is currently working as research associate at the Center for Broadband Telecommunications. His fields of interest are quantum-well devices and highspeed optical transmission systems.

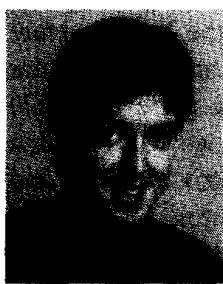

Niels Storkfelt (S'93) was born in Copenhagen, Denmark, in August 1965. He received his M.S. degree in electrical engineering from the Electromagnetics Institute, Technical University of Denmark (DTU) in 1990. He is now a Ph.D. student at the Center for Broadband Telecommunications, where he is conducting research in optical amplifiers and their applications as functional devices.

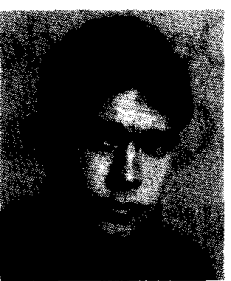

Michael Vaa was born in Copenhagen, Denmark, on February 23, 1966. He received the M.Sc. degree in electronic engineering at the Technical University of Denmark (DTU) in 1991.

In 1990 he spent five months at NEC Optoelectronic Device Research Laboratory working with MQW DFB semiconductor laser diodes. Since 1991 he has been working as a research associate at the Center for Broadband Telecommunications, DTU. His current research interests are in the field of optical high-speed signal generation and processing, with emphasis on semiconductor optical amplifiers.

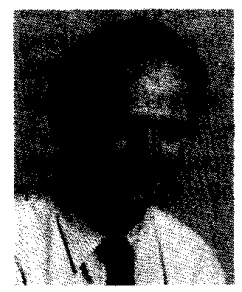

Kristian E. Stubkjaer (S'76-S'78-S'80-M'81) was bom in Denmark in 1953 . He received the M.Sc. and Ph.D. degrees from the Technical University of Denmark (DTU), Copenhagen, in 1977 and 1981, respectively. From 1979 to 1981 he studied at the Tokyo Institute of Technology, Tokyo, Japan, with a scholarship from the Japanese Government.

From 1981 to 1982 he was drafted for military service at the Danish Defence Research Establishment in Copenhagen. From 1982 to 1983 he was a visiting scientist at the IBM T. J. Watson Research Center, Yorktown Heights, NY. He is now an associate professor at the Technical University of Denmark (DTU), where he is working in the field of optical communication. From 1985 to 1990 he was director of the Electromagnetics Institute, and currently he is serving as chairman for the Electrotechnical Committee under the Technical Research Council (Danish Ministry for Education). 